Impfungen - viele Fragen, unterschiedliche Einschätzungen

Störend am Artikel mit der Replik von Frau Sommaruga und Frau Bachmann ist die Tatsache, dass sie implizieren, die Dres. Hirte/Albonico seien als «Impfexperten» mehr berufen als zum Beispiel die grossmehrheitliche schweizweite Anzahl praktizierender Fachärztinnen/-ärzte für Kinder- und Jugendmedizin (über 90\%), die ihre Klientel (Eltern und Patienten) mindestens ebenso differenziert beraten - auch in allen Impffragen - wie die Autoren des Impfratgebers SKS. Das Forum für Praxispädiatrie mit über 360 Praxispädiatern/-innen verfügt insgesamt über deutlich mehr «Know-how» als die zwei selbsternannten «Spezialisten» und steht deren Ausführungen im Ratgeber SKS - übrigens seit Beginn an - äusserst kritisch gegenüber.

Dr. med. P. Reinhard, Kloten

\section{Zur Impfdiskussion}

Als Allgemeinpraktikerin schätze ich den Impfratgeber der SKS seit nun fünf Jahren. In meiner Praxis betreue ich viele sehr impfkritische Eltern. Mit Hilfe des Impfratgebers kann ich sie überzeugen, wenigstens die wichtigsten Impfungen bei ihren Kindern durchführen zu lassen, also z. B. Tetanus und Polio sowie Röteln bei Schulabgängerinnen. Der Impfratgeber nimmt alle Bedenken dieser Eltern ernst und erlaubt ein Abwägen der Risiken, welches die Eltern dann bewusst und in Eigenverantwortung übernehmen können. Leider sind zunehmend weniger Einzelimpfstoffe verfügbar, so dass viele dieser Eltern nicht mehr zum Impfen zu überzeugen sind. Wenigstens gibt es Tetanus noch als Einzelimpfstoff.

\section{Dr. med. Danielle Lemann, Langnau}

\section{Impfartikel in der Ärztezeitung Nr. 9/2005}

Ich bin sehr dankbar für den ausführlichen Artikel von C.-A. Siegrist, C. Aebi, D. Desgrandschamps, U. Heininger und B. Vaudaux zum Impfratgeber der Stiftung für Konsumentenschutz. Insbesondere, da die Pro Juventute einen ähnlichen «Kritischen Impfführer» herausgegeben hat von Beat W. Hollenstein und Thomas Pfluger. Dort stehen die gleichen unfundierten Meinungen als gute Empfehlungen an Eltern drin.

Ich bin froh, dass wir jetzt eine Zusammenstellung von Fakten mit Literaturangabe haben, mit denen wir die Behauptungen der Impfgegner besser als bisher widerlegen können.

Das Wichtigste aber wäre (und das hoffe ich), dass die Impfgegner unter den Ärzten und die sogenannt «impfkritischen» Ärzte diesen Artikel mindestens lesen, bevor sie ihre Klienten weiterhin im alten Sinne beraten (wenn sie es nach dem Lesen wirklich noch verantworten können, die Beratung auf die alte Weise weiterzuführen).

Dr. med. Katharina Takken-Sahli, Fällanden

\section{Impfratgeber}

Ich finde es absolut grossartig, ja endlich an der Zeit, dieser unsäglichen SKS-Broschüre mit Evidenz zu begegnen. Diese wird nämlich in der täglichen Praxis von impfkritischen Eltern sehr oft und gerne zitiert.

Ich möchte anregen, dass dies nicht nur in der SÄZ, d.h. für Fachkreise, sondern auch breit in der Laienpresse gestreut wird. Nur durch intensive, objektive, mit Evidenz und nicht mit Angstmache, sich immer wiederholender Information können Eltern unserer Patienten und andere zweifelnde Kreise für unser Anliegen zum Wohle der von uns betreuten Kinder überzeugt werden.

Dr. med. Thomas Bamberger, Grenchen

\section{Impfungen}

Herzlichen Dank für die Veröffentlichung des Interviews und des Impfratgebers. Einerseits werden viele der verbreiteten Missverständnisse geklärt, als klinischer Infektiologe werden mir dieselben Fragen sowohl von praktizierenden Ärzten, von Medizinalpersonen im Spital, aber auch von sogenannten «Laien» gestellt. Hartnäckig halten sich Vorurteile wie «Überforderung des Immunsystems» wie auch «Schwächung des Organismus wegen fehlender Infektionserfahrung».

In den Diskussionen erlebe ich immer wieder, dass selbst Medizinalpersonen nicht unterscheiden können zwischen Schutz eines Individuums und einer Population, die Bedeutung der Herdenimmunität ist oft nicht bekannt. Jedes Ereignis nach einer Impfung wird als deren Folge interpretiert: Die Unterscheidung zwischen Kausalität und Koinzidenz scheint zu mühsam zu sein.

Die Persistenz solcher Ideen hängt vermutlich damit zusammen, dass wir Gerüchten lieber Glauben schenken als nüchternen Tatsachen. Ich möchten den Autoren des «Impfratgebers» 
vor allem für diese ausführliche und sachliche Widerlegung der Argumente in der SKS-Broschüre danken. Gut, dass endlich jemand Klartext gesprochen hat!

Dr. med. Marco Rossi, Luzern

\section{Artikel von Claire-Anne Siegrist in der Ärztezeitung}

Als Vertreter der Elternschaft und tätig im Gesundheitswesen möchte ich Prof. Claire-Anne Siegrist und den Experten von InfoVac für den äusserst interessanten und aufklärenden Artikel gratulieren.

Ich teile die Meinung der InfoVac-Experten und kann nur bestätigen, dass die Entwicklung in unseren Nachbarländern Frankreich und Italien sowie in Belgien, Portugal, Spanien, aber auch in Grossbritannien, Schweden, den USA, Mexiko und Brasilien den durch die Behörden und Eltern erkannten Stellenwert einer fortschrittlichen nationalen Präventions- und Impfstrategie bestätigen.

Drei Faktoren fallen mir dabei auf:

1. Es ist stark zu begrüssen, dass auf diesem Gebiet immer mehr internationale Interessengruppen sich auf wissenschaftliche, evidenzbasierte Aussagen abstützen.

2. Wir erleben in vielen Ländern, dass der z.T. rasch ansteigende Bedarf von Impfstoffen bei Epidemieausbruch vielfach nicht bis ungenügend abgedeckt werden kann, da es sich hier um äusserst komplexe zeitaufwendige Produktionsverfahren handelt. Eine effiziente Prävention muss frühzeitig geplant sein und kann nur schwerlich aus einer «Schlusslichtposition» gemeistert werden. (Masern)

3. Der frühzeitigen, effizienten Aufklärung/ Kommunikation zwischen Behörden, Experten, Ärzten und Eltern kommt für eine effiziente nationale Impfung und Schutz der Bevölkerung eine entscheidende Rolle zu.

Ich möchte die Impfverantwortlichen in diesem Lande ermutigen, die notwendigen Massnahmen zu treffen, dass der Schutz der Bevölkerung und speziell der Kinder in der Schweiz weiter verbessert werden kann.

Jorge Wernli, Zug

\section{Kommentar zum Artikel über Impffragen}

Hervorragende Analyse und Kritik der irreführenden und pseudoausgewogenen Broschüre des Konsumentinnenforums! Diese Fehler selber aufzudecken sprengt die zeitlichen Möglichkeiten eines Grundversorgers.

Dr. med. Res Kielholz, Uster

\section{InfoVac}

Ich teile die Meinung, welche in der Ärztezeitung durch InfoVac-Experten vertreten wird. Gerade in Zeiten grosser Migration sind wir auf eine vernünftige Impfberatung basierend auf Fakten angewiesen! Als Kinderärztin schätze ich die fundierten und kompetenten Informationen des InfoVac-Teams sehr.

S. Isay-Utzinger, Basel

\section{SÄZ-Kontroverse SKS-Impfratgeber}

Sie, Frau Kollegin C.-A. Siegrist, fordern ja flott heraus mit Ihrem Frühlingsputz des Impfratgebers der Stiftung für Konsumentenschutz SKS. Da ich bereits das Vergnügen hatte, Sie persönlich kennenzulernen, verwundert mich Ihr energisches und gründliches Vorgehen nicht. Ich möchte Sie nun auffordern, mit der gleichen Gründlichkeit die bisherige Informationstätigkeit des BAG in Sachen Routineimpfungen des Kindesalters zu analysieren und das Ergebnis detailgetreu der Leserschaft der SÄZ zugänglich zu machen.

Im Interview (Seite 535) fordern Sie ja mit Recht ein partnerschaftliches Vorgehen im Rahmen der Impfpromotion. Ich bin froh, dass Sie, nun als Präsidentin der Eidgenössischen Kommission für Impffragen, dabei mit so deutlichen Worten Folgendes feststellen: «Die Qualität und Quantität der den Ärzten und Patienten gelieferten Informationen war leider lange ungenügend. Dies ist übrigens heute noch so und bietet Angriffsflächen, [...].» Es ist genau diese bedauernswert insuffiziente und gelegentlich auch irreführende Information, mit der von offizieller Seite sowohl die Familienärzte wie auch die betroffenen Eltern seit 1987 beglückt werden, welche mich schon lange verwundert und auch ärgert.

Sie fahren im Interview dann fort: «[...] und bietet Angriffsflächen, die dann zum Beispiel in der von der SKS vertriebenen Broschüre genutzt werden.» Sind Sie hier nicht ein bisschen voreilig? Man könnte die Geschichte ja auch andersherum lesen, etwa so: Das Zielpublikum der Impfpromotion sind potentielle Konsumenten von gesundheitlichen Vorsorgeleistungen. Diese haben das Recht auf umfassende und unabhängige Information. Die SKS hat mit guten Gründen befunden, dass diese Bedingung in Sachen Kinderimpfungen nicht erfüllt ist, und hat, ganz im Sinne ihres Auftrages, dem interessierten Publikum ergänzende Informationen zur Verfügung gestellt. Dies ist in meinen Augen in keiner Weise ein destruktives Ausnutzen der diagnostizierten Schwächen.

Wir sind uns wohl einig, es gibt seit bald 20 Jahren in dieser Sache Handlungsbedarf: Die SKS hat 
gehandelt, Sie beginnen zu handeln. Sie haben nun die Gelegenheit, Ihre Unabhängigkeit unter Beweis zu stellen und die Analyse auf der Verursacherseite zu vervollständigen. Mit Spannung erwarte ich Ihren Bericht.

Dr. med. Peter Klein, Bern

\section{Impfratgeber: Fortsetzung!}

Mit grossem Interesse habe ich Ihren sehr differenzierten Bericht bzw. die Stellungnahme zu diesem doch sehr heiklen Thema gelesen und teile Ihre Meinung weitgehend.

Immer wieder sehe ich mich als pädiatrischer Grundversorger diesen Fragen ausgesetzt und realisiere, dass man gewissen Leuten die Wichtigkeit der Impfung nicht näherbringen kann. Und da schliesslich die Entscheidung und somit auch die Verantwortung direkt bei den Eltern liegt - natürlich nach gründlicher Impfaufklärung -, sind mir häufig die Hände gebunden. Diese Ohnmacht beschränkt sich glücklicherweise auf ein paar wenige Patienten, dennoch scheinen die schützbaren und z.T. schweren Krankheiten zunehmend in Vergessenheit zu geraten, und eines scheint mir sicher ... das Pendel schlägt um. Am ehesten zeigt sich dies bei mir bei den MMR-Impfungen.

Dank Ihrer fundierten Berichte werden mir handfeste Werkzeuge mitgegeben, um noch besser zu informieren und zu argumentieren. Dennoch werden wir einige Leute, die anscheinend gänzlich abgelöst von den schulmedizinischen Ansichten erscheinen, nie erreichen. Herzlichen Dank für Ihre wertvolle Arbeit

Dr. O. Zerwetz, Egg/ZH

\section{Impfratgeber}

Die klare und fundierte Stellungnahme der Präsidentin der eidgenössischen Impfkommission ist sehr zu begrüssen und verdient die uneingeschränkte Unterstützung der Ärzteschaft und ihrer Gremien. Im Gegensatz zu den in letzter Zeit in der Schweizerischen Ärztezeitung vehement diskutierten komplementärmedizinischen Massnahmen, bei denen es letztlich um individualmedizinische Entscheide mit höchstens finanziellen gesellschaftlichen Folgen (und Interessen) geht, ist die Verhütung ansteckender Krankheiten mit rational begründeten Methoden seit der Aufklärung eine der nobelsten gesellschaftlichen Aufgaben der Ärzteschaft. Zwar ist es in Zeiten des grassierenden Individualismus unpopulär bzw. in gewissen Kreisen gar politisch unkorrekt, darauf hinzuweisen, dass zum Wohle der Gesellschaft als Ganzem individuelle Ansprüche in den Hintergrund zu treten haben trotzdem: Impfgegner profitieren parasitär vom Impfherdenschutz, den sie durch ihre letztlich egoistische Haltung gleichzeitig gefährden.

Leider werden sich die wenigsten Impfgegner durch rationale und wissenschaftlich fundierte Argumente umstimmen lassen - sie werden höchstens ihre Kinder notfallmässig gegen Masern impfen lassen wollen, wenn die Ferien in Italien durch eine dort grassierende Masernepidemie gefährdet sind.

Dr. med. J. Gubler, Zürich

\section{Impfratgeber: Evidenz anstelle von Behauptungen}

Ich teile voll und ganz die Meinung der InfoVacExperten, die im oben erwähnten Artikel in der Schweizerischen Ärztezeitung zum Ausdruck kommt. Es ist äusserst bedenklich, dass Eltern durch Fehlinformationen, Halbwahrheiten und Lügen ihre Kinder und deren Umgebung unnötigen Risiken, d.h. durch Impfungen verhütbare Krankheiten, aussetzen. Die Stiftung für Konsumentenschutz müsste bezüglich dem «Rategeber: Impfen - Grundlagen für einen persönlichen Impfentscheid» besser in Stiftung für Konsumentengefährdung umbenannt werden.

Dr. med. Oliver Adam, Solothurn

\section{Impffragen}

Ich teile die Meinung der Impfexperten in der Schweizerischen Ärztezeitung, und ich bin sehr enttäuscht, dass sich die Konsumentenorganisation für so tendenziöse Aussagen einspannen lässt. Man müsste zum Beispiel die Zulassung der Homöopathen davon abhängig machen, dass sie sich nicht mit falschen Argumenten gegen Impfungen äussern dürfen.

Dr. med. C. Willi, Illnau

\section{Prise de position du groupe InfoVac}

Par ce message, j'aimerais exprimer mon accord avec les opinions publiées par les experts d'InfoVac, et les remercier de la qualité de leur travail. J'apprends que vous ouvrez vos colonnes à un débat au sujet de leur prise de position sur la brochure de la SKS. Encourager ce débat me semble une attitude ambiguë. En effet, les arguments développés par la SKS ne s'encombrent généralement pas de probité intellectuelle. Face à certains 
mensonges avérés, la FMH ne devrait-elle pas défendre les opinions du groupe InfoVac de manière plus explicite?

Giorgio Zanetti, Lausanne

\section{Impfratgeber: Evidenz anstelle von Behauptungen}

Für Ihre grosse Arbeit und der Zurverfügungstellung von objektiven Informationen möchte ich Ihnen - sicher auch im Namen vieler betroffenen und verunsicherten Familien - ganz herzlich danken, ich kann mir vorstellen, wie gross Ihr Zeitbedarf für die Erstellung dieser Arbeit gewesen ist.

Schön wäre es, wenn Ihr Argumentarium bei Gelegenheit in einer handlichen Broschüre (gleiche Grösse wie der Impfratgeber !) zur Verfügung gestellt werden könnte, damit es interessierten Eltern auch abgegeben werden kann.

\section{Ryffel, Münchenbuchsee}

\section{InfoVac}

Je partage les opinions exprimées par les experts d'InfoVac dans le Bulletin des médecins suisses et je suis toujours très curieuse de lire le nouveau bulletin. Il est facile à lire, synthétique et très pratique et utile surtout dans notre domaine au CICR.

Dr Jacqueline Avril, Genève

\section{Guide sur les vaccinations}

J'ai lu avec grand intérêt le texte publié par les experts d'InfoVac dans le Bulletin des médecins suisse. Je les remercie pour leur travail et leur approche scientifique, basée sur la médecine fondée sur les preuves. Il s'agit là d'un travail important, méritant une large diffusion auprès des professionnels, mais également auprès du public. Je partage les opinions exprimées par les experts d'InfoVac dans le Bulletin des médecins suisses.

Dr René Tabin, Sion

\section{Impfstellungnahme von Prof. Siegrist}

Ich bin Prof. Siegrist für die klaren Aussagen zum Thema Impfen sehr dankbar und teile diese Statements aus meiner Sicht voll und ganz.

Dr. med. Mathias Wenger, Frauenfeld
Publication des recommandations pour vaccins en Suisse et interview du Prof. C.-A. Siegrist

Je salue l'initiative de l'OFSP et du Bulletin des médecins suisses de rendre publique la position des experts concernant la vaccination en Suisse. Les explications apportées sont claires, objectives, prenant en compte les interrogations voire les peurs des adversaires de la vaccination, et tout en partageant bien entendu l'avis des experts, j'espère que cela contribuera à plus de sérénité dans le débat sur la vaccination. J'espère également que la phrase sibylline insérée en fin du commentaire par l'OFSP disant que les propositions du groupe d'experts pourraient ne plus être suivies à l'avenir ne soit qu'un mirage dans le désert et non pas le signe avant-coureur d'un tsunami médico-social assez inquiétant.

Dr Jean-Luc Magnenat, Carouge

\section{Artikel betr. Impfen}

Ich bin InfoVac sehr dankbar, dass eine derart klare und eindeutige Stellungnahme veröffentlicht wurde. Gerade auch von seiten eines Kantonsarztes eines kleinen Kantons kann nicht genug darauf hingewiesen werden, wie wichtig eine genügende Durchimpfung der Bevölkerung für die Volksgesundheit generell ist.

Dr. med. Daniel Brunner, Ennenda

\section{Vaccination}

Pédiatre, devant régulièrement défendre l'utilité des vaccins j'ai beaucoup apprécié l'article de Madame le Professeur Siegrist sur la vaccination et sa critique des détracteurs de celle-ci. Son article m'est fort utile pour discuter avec les parents. Bien qu'ayant acquis une formation en homéopathie et reconnaissant son intérêt je ne peux cautionner la critique sans fondement que ses partisans font des vaccins.

Je remercie donc vivement les membres d'InfoVac de leur travail.

M. Giordano Le Locle

\section{Impfartikel}

Ich möchte meine Zufriedenheit über den Artikel des InfoVac-Teams ausdrücken. Die aktuell kompetenteste Gruppe hat zu einem Thema, in dem allzu viele Unkorrektheiten publiziert wer- 
den, klar und deutlich Stellung bezogen. Dabei geht es nicht um Polarisierung, sondern um präzise Information.

Dr. U. Bühlmann, Zürich

\section{Guide sur les vaccinations}

Quelques infirmières de mon service m'ont demandé quel était le ton de l'article, car elle semblaient connaître vos (fortes) convictions. J'ai répondu que le ton était tout sauf sectaire, et que les preuves avancées sont, pour un médecin formé dans nos universités, parfaitement correct, utile, et accessible.

\section{Dr Virgile Woringer, Lausanne}

\section{InfoVac-Ped \& Artikel}

Bezugnehmend auf die umfangreiche Dokumentation und Replik der InfoVac-Experten auf die unsäglich tendenziöse Konsumenten-Informationsschrift «Impfratgeber» möchte ich hiermit meinen Dank an die Autoren aussprechen und mein volles Einverständnis mit dem Inhalt der Replik ausdrücken.

Leider ist der Schaden auf die primäre und sekundäre Präventionsarbeit der pädiatrischen Grundversorgung durch diese Impfbroschüre gesetzt und in den nächsten Jahren nicht wieder gutzumachen: die Durchimpfungsrate ist im Sinken begriffen und wird weiter sinken. Es müssten unverhältnismässig grosse Anstrengungen gemacht werden, um die WHO-Ziele zu erreichen! Auf ein pikantes Detail wurde bisher noch nie eingegangen: Wie und warum war es möglich, dass die FMH diese Impfbroschüre mit einem namhaften Betrag unterstützt hatte? Wie wird die FMH in Zukunft ähnliche Projekte behandeln? Gibt es einen Kontrollmechanismus innerhalb der FMH, welcher gewährleistet, dass ein Fachgremium angehört werden muss, bevor Gelder für solch schädigende Informationsschriften gesprochen werden? Im vorliegenden Falle hätten wohl die SKIF-Experten, die Fachgesellschaft SGP und der Berufsverband Forum für Praxispädiatrie angefragt werden sollen!

Dr. med. P. Trefny, Luzern

\section{Impfratgeber}

Ich teile die Meinung der InfoVac-Experten/-Expertin betreffend dem SKS-Impfratgeber gar nicht. Ich erwarte von sogenannten Experten, welche auch alle Akademiker sind, mehr Eigenkritik und eine Bereitschaft, sich mit Kritik ernst- haft auseinanderzusetzen. Vielleicht müssten sich diese Kolleginnen und Kollegen einmal überlegen, weshalb eine solche Impfmüdigkeit entstanden ist. Nur die Kritiker, welche sich sehr viele Gedanken zur ganzen Impfgeschichte machen, versuchen, schlechtzumachen, damit ist das Problem nicht gelöst. Diese Berichte haben $\mathrm{zu}$ einer weiteren Polarisierung beigetragen. Ist das Ihr Ziel?

\section{Dr. med. Aurelio Nosetti-Bürgi, Emmenbrücke}

\section{Impfungen}

Endlich reagiert die Ärzteschaft auf die jahrelangen Versuche, das ehrgeizige Impfprogramm des BAG vom 11. November 2002 zu diskreditieren. Als Grundversorger entsteht oft das Gefühl, mit dem Rücken zur Wand zu stehen gegenüber «kritischen» Äusserungen von Patienten oder selbsternannten Patientenvertretern. Für eine Minderheit von Menschen ist a priori alles suspekt, was von offizieller Seite (BAG, FMH, Fachgesellschaften) in der Prävention empfohlen wird. Die meisten «Impfkritiker» unter meinen Patienten sind mangelhaft orientiert und auch sachlichen Argumenten nicht zugänglich. Es geht da um Glaubensfragen. Es ist nun an der Zeit, dass «Sektierer» unter den Ärzten konfrontiert werden mit Aussagen, die nachgewiesenermassen unwahr sind und in unfairer Weise immer wieder unter die Leute gebracht werden. Um so wichtiger für uns Hausärzte sind offizielle Stellungnahmen von Experten und ein sanfter Druck auf die «Konsumenten», Eigenverantwortung zu übernehmen. Die Argumente gegen die offizielle Impfstrategie gründen auf archaischen Ängsten, die schon zur Zeit von Jenners Kuhpockenimpfung kursierten. Ähnliche Argumente wurden von Gegnern auch gegen das Sicherheitsgurtenobligatorium vorgebracht, gegen Tempobeschränkungen usw.

Ich danke den Impfexperten von InfoVac für die kompetente und fortlaufende Orientierung von uns Grundversorgern.

Dr. med. Marcel Bühler, Brütten

\section{Guide sur les vaccinations: suite!}

En réaction à l'article du Prof. Claire-Anne Siegrist, je déclare sans ambages partager les opinions exprimées par les experts d'InfoVac! Je trouve heureux et très opportun ce type de mise au point. En tant qu'obstétricien je diffuserai cet article à mes patientes intéressées!

Dr Andrei Cepleanu, Epalinges 